\title{
Editorial
}

\section{Social mechanisms of robot programming by demonstration}

\section{Special issue composition}

The special issue collects a subset of the best papers presented at the ICRA'05 workshop on the Social Mechanisms of Robot Programming by Demonstration. The workshop took place on April 21st 2005 in Barcelona, Spain, at the IEEE Conference on Robotics and Automation (ICRA'05). Eleven of the best papers presented at the workshop were subsequently submitted for peer review before publication in the special issue, eight of which were then selected for publication.

The special issue emphasizes the role that social mechanisms play in driving and guiding robot programming by demonstration (RbD). It is a follow-up on our 2004 special issue on "Robot Programming by Demonstration" of the Robotics and Autonomous Systems Journal [2].

\section{Motivation}

Programming by demonstration $(\mathrm{PbD})$ has become a central topic of robotics, which spans across general research areas such as human-robot interactions, machine learning, machine vision, and motor control. The field was started by combining classical engineering approaches, typically for controlling redundant manipulator arms, with artificial intelligence techniques which allow for planning in a blockworld type of task representation. Incrementally, the field adopted an interdisciplinary approach, drawing from the neurosciences and social sciences, taking into account evidence of specific neural mechanisms for visuo-motor imitation in primates $[5,3]$ as well as the developmental stages of imitation capacities in children [7,6]. Eventually, the notion of "Robot Programming by Demonstration" was replaced by the more biological label of "Imitation Learning".

This special issue reflects the strong interdisciplinary approach of the field, focusing on the social skills that Robot $\mathrm{PbD}$ requires, as well as contributes.

One of the core ideas of developmental psychology is that Imitation Learning is goal-directed; actions are meant to fulfill a specific purpose and convey the intention of the actor. While a long-standing trend in $\mathrm{RbD}$ has been to approach the problem from the standpoint of following trajectories and replicating joint motions (see Aleotti and Caselli and Ogino et al., this issue), the majority of the works presented in this special issue start from the assumption that imitation is not just about observing and replicating the motion, but rather about understanding the goals of a given action. Learning to imitate relies importantly on the imitator's capacity to infer the demonstrator's intentions (see Erlhagen et al., this issue). However, demonstrations may be ambiguous and extracting the intention of the demonstrator requires the construction of a cognitive model of the demonstrator (see Jansen and Belpaeme and Chella et al., this issue), as well as the use of other social cues to provide complementary knowledge (see Breazeal et al., this issue).

Understanding the goal of the task is still only half of the picture, as there may be several ways in which the goal may be achieved. Moreover, what is good for the demonstrator may not necessarily be good for the imitator. Thus, different models may be allowed to compete in order to find a solution that is optimal both from the point of view of the imitator and that of the demonstrator (see Demiris and Khadouri, this issue).

Finally, Robot $\mathrm{PbD}$ is about learning skills. At its core it must be generic in its representation of the skills and in the way it generates them, so that the imitator can adapt its imitation to different contexts and to different embodiments to that of the demonstrator (see Billard et al., this issue).

\section{Links}

The reader may be interested in two upcoming special issues that address topics complementary to Robot Programming by Demonstration.

Billard and Schaal's special issue on the "Brain Mechanisms of Imitation Learning", to appear in April 2006 in Neural Networks [1] will review computational models of the neural mechanisms underlying imitation learning in animals and their application to controlling robots. This special issue will cover works that are inherently biological in their approach and that provide hypotheses for further neurological and psychological studies of imitation in animals.

Demiris and Billard's special issue on "Robot Learning by Observation, Demonstration, and Imitation", to appear in August 2006 in the IEEE Transactions on Systems, Man and Cybernetics $B$ journal [4] will put forward novel learning techniques applied to solving $\mathrm{RbD}$, from the perception and recognition of actions through to their reproduction. 


\section{References}

[1] A. Billard, S. Schaal, Special issue on the brain mechanisms of imitation learning, Neural Networks 19 (4) (2006).

[2] A. Billard, R. Siegwart, Special issue on robot programming by demonstration, Robotics and Autonomous Systems 47 (2004) 2-3.

[3] J. Decety, T. Chaminade, J. Grezes, A.N. Meltzoff, A pet exploration of the neural mechanisms involved in reciprocal imitation, Neuroimage 15 (2002) 265-272.

[4] Y. Demiris, A. Billard, Special issue on robot learning by observation, demonstration and imitation, IEEE Transactions on Systems, Man and Cybernetics Part B (August) (2006) (in press).

[5] G. Rizzolatti, L. Fadiga, L. Fogassi, V. Gallese, Resonance behaviors and mirror neurons, Archives Italiennes de Biologie 137 (2-3) (1999) 85-100.

[6] J. Nadel, C. Guerini, A. Peze, C. Rivet, The Evolving Nature of Imitation as a Format for Communication, Cambridge University Press, 1999, pp. 209-234.

[7] J. Piaget, Play, Dreams and Imitation in Childhood, Norton, New York, 1962.
Aude Billard*

LASA Laboratory, School of Engineering, Ecole Polytechnique Federale de Lausanne - EPFL, Station 9, 1015-Lausanne, Switzerland E-mail address: aude.billard@epfl.ch.

Ruediger Dillmann

Forschungszentrum Informatik, IDS, University of Karlsruhe, Haid-und-Neu-Str. 10-14, 76131 Karlsruhe, Germany

Available online 13 March 2006

* Corresponding editor. Tel.: +41 21693 5464; fax: +41 21693 7807. 